\title{
ANOMALOUS SKIN-EFFECT IN TIN CYLINDERS
}

\section{L.J.M. Van de KLUNDERT, E.A. GIJSBERTSE and L.C. Van der MAREL Twente University of Technology, Enschede, The Netherlands}

\author{
Received 14 June 1972
}

\begin{abstract}
The susceptibilities of three $\mathrm{Sn}$-cylinders have been measured at a temperature slightly below $T_{\mathrm{c}}$ and in an external magnetic field just below $H_{\mathrm{c}}(T)$. The results are compared with calculations for a flat plate. From this the d.c. conductivity, the mean free path and the reflectivity-factor, have been determined.
\end{abstract}

Measurements are performed on three Sn-cylinders: a polycrystalline one (I) and two single crystals with the $c$-axis parallel (II) and perpendicular (III) to the cylinder-axis respectively. The equipment used in these experiments is the same as already described $[1,2]$. The complex susceptibility was measured in the frequency range $4.4200 \mathrm{~Hz}$, at a temperature just below $T_{\mathrm{c}}$ and an external magnetic field just above $H_{\mathrm{c}}(T)$.

The behaviour can be described in terms of the extended law of Ohm

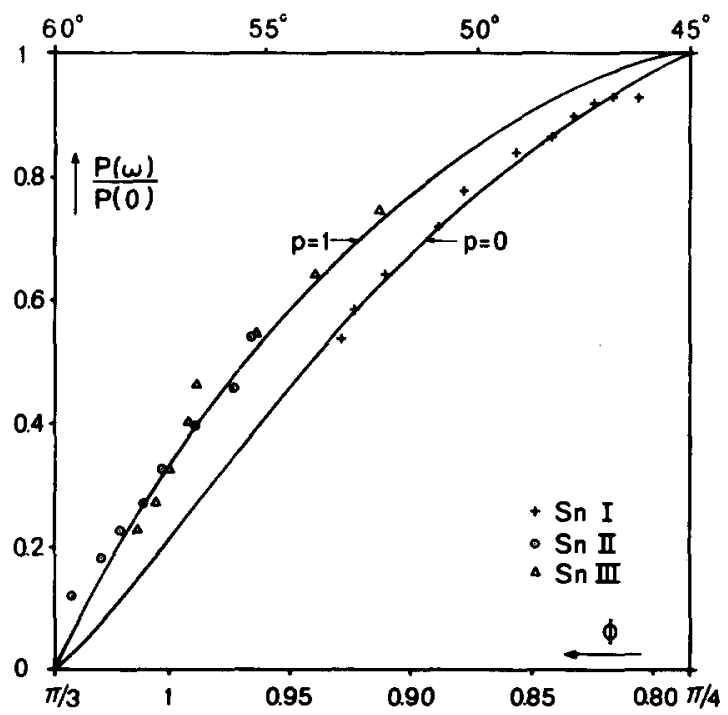

Fig. 1. The dimensionless quantity $P(\omega) / P(0)$ as a function of $\phi(\omega)$ for a flat surface according to Dingle [3] for $p=0$ and $p=1 ; \phi$ is indicated in radians as well as in degrees.
$J(r)=\int_{0}^{a} f\left(\sigma_{0}, a, \lambda, p, r, r^{\prime}\right) \cdot E\left(r^{\prime}\right) \mathrm{d} r^{\prime}$

$\sigma_{0}$ is the d.c. conductivity, $a$ the radius of the cylinder, $\lambda$ the mean free path and $p$ the reflectivity-factor. With $f=\sigma_{0} \delta\left(r-r^{\prime}\right)(1)$ becomes the well-known form of Ohm's law. Calculations with respect to (1) for a flat plate have been performed by Dingle [3].

The values of $\chi^{\prime}$ and $\chi^{\prime \prime}$ can be transformed to values of $\rho$ and $\phi$ according to [1]

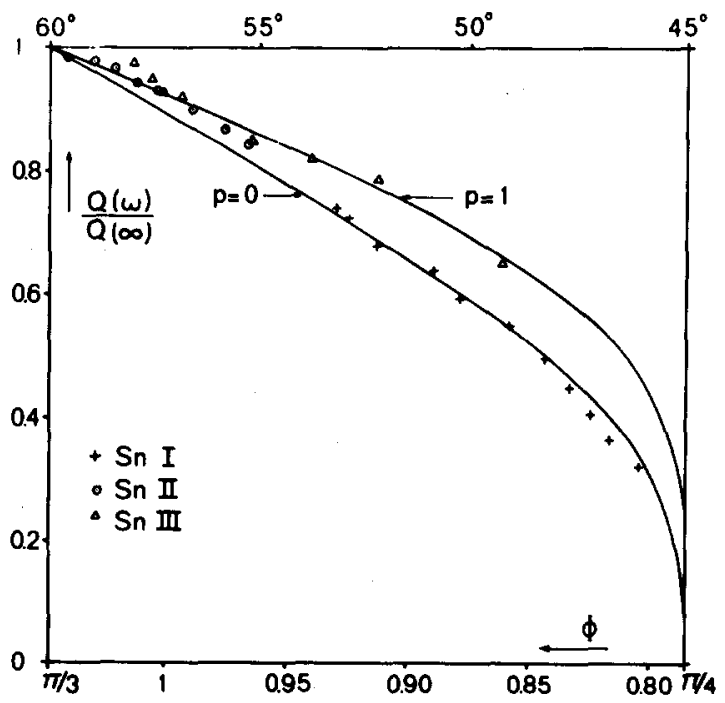

Fig. 2. The dimensionless quantity $Q(\omega) / Q(\infty)$ as a function of $\phi(\omega)$ for a flat surface according to Dingle [3] for $p=0$ and $p=1$. 
Table 1

\begin{tabular}{lccc}
\hline sample & Sn I & Sn II & Sn III \\
\hline radius $(\mathrm{mm})$ & 2.50 & 2.90 & 2.75 \\
length $(\mathrm{mm})$ & 20.0 & 20.1 & 24.3 \\
purity & $5 \mathrm{~N}$ & $6 \mathrm{~N}$ & $6 \mathrm{~N}$ \\
$p$-value & 0 & 1 & 1 \\
$\sigma_{0}\left(10^{10} \Omega^{-1} \mathrm{~m}^{-1}\right)$ & 5.8 & 54 & 27 \\
$\sigma_{0} / \lambda\left(10^{14} \Omega^{-1} \mathrm{~m}^{-2}\right)$ & 5.7 & 8.1 & 8.1 \\
$\lambda\left(10^{-3} \mathrm{~m}\right)$ & 0.1 & 0.6 & 0.3 \\
$\nu_{0}($ from graph $)(\mathrm{Hz})$ & 260 & 0.16 & 1.34 \\
$\nu_{0}($ from table $)(\mathrm{Hz})$ & 278 & 0.72 & 5.81 \\
\hline
\end{tabular}

$\chi=\chi^{\prime}+\mathrm{j} \chi^{\prime \prime}=J_{2}(a k) / J_{0}(a k)$

with $a k=\rho \exp (\mathrm{j} \phi) ; k$ is related to the complex skin depth $\delta$ by $k=\mathrm{j} / \delta$. Our experimental values of $\rho$ and $\phi$ are compared with those calculated from Dingle's table II [3]. Dingle considered only two values of $p$ : $p=0$ diffuse reflection, $p=1$ specular reflection. Independently of $p$ the d.c. conductivity $\sigma_{0}$ is given by

$\sigma_{0}=P(0) / \mu_{0}$ with $P(0)=\lim _{\omega \rightarrow 0} \rho^{2}(\omega) / a^{2} \omega$.

$\sigma_{0} / \lambda$ can be calculated according to the formulae of Reuter and Sondheimer [4] which depend on $p$ :

$$
\begin{array}{ll}
p=0: & \sigma_{0} / \lambda=\sqrt{3} Q(\infty) / 2 \pi \mu_{0}, \\
p=1: & \sigma_{0} / \lambda=729 \sqrt{3} Q(\infty) / 1024 \pi \mu_{0} .
\end{array}
$$

with $Q(\infty)=\lim _{\omega \rightarrow \infty} \rho^{3}(\omega) / a^{3} \omega$. Dingle's results have been plotted in three different ways in the figs. 1,2 and 3 together with our experimental data. The value of $p$ was chosen on account of these figures. For sample I, $p=0$ appeared to be a good approximation as expected; for the samples II and III, however, $p=1$ seems to be a better value. In the figs. 1 and 2 , $P(\omega) / P(0)$ and $Q(\omega) / Q(\infty)$ have been plotted as a function of $\phi$ respectively. The values of $P(0)$ and $Q(\infty)$ can be obtained from these figures, so the values of $\sigma_{0}, \sigma_{0} / \lambda$ and $\lambda$ can be calculated. The results are given in table 1 . The obtained values of $\sigma_{0} / \lambda$ agree quite well with the results of Lyall and Cochran [5]. The low value of $\sigma_{0} / \lambda$ for sample I can be explained by surface-effects [6] due to the preparation. To check the validity of the values of $\sigma_{0}$ and $\lambda$ so far ob-

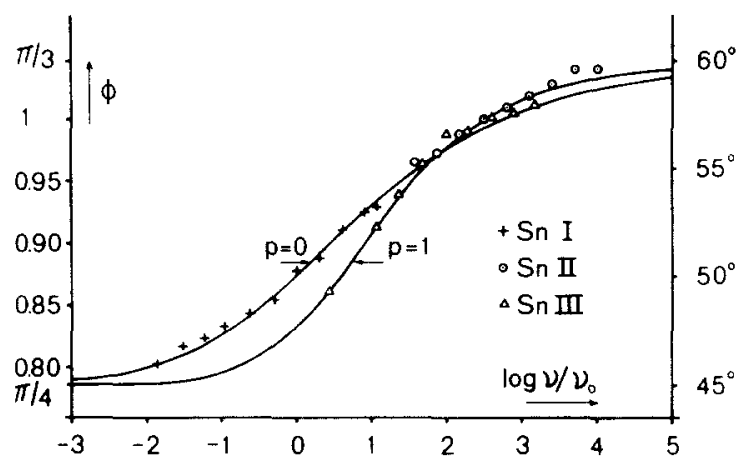

Fig. 3. $\phi(\nu)$ as a function of $\log \left(\nu / \nu_{0}\right)$ according to Dingle [3] for a flat surface.

tained $\phi$ has been plotted as a function of $\log \nu$ (see fig. 3).

According to Dingle a scaling factor $\nu_{0}$

$\nu_{0}^{-1}=\frac{3}{2} \pi \mu_{0} \sigma_{0} \lambda^{2}$

has to be introduced. By putting our results as good as possible on Dingle's figure also a graphical value of this scaling factor can be determined. In the case of sample I the values of $\nu_{0}$ agree quite well. For the samples II and III, however, there is a rather large difference. The values are compiled in table 1. Possibly this discrepancy is also due to the fact that the experimental data, obtained on cylinders, are compared with Dingle's results for a flat plate. To show the influence of the geometry of the sample calculations using the exact shape of the function $f$ in (1) are in progress and will be published.

\section{References}

[1] L.J.M. Van de Klundert, A. van Ardenne and L.C. van der Marel, Phys. Lett. 34A (1971) 321.

[2] L.J.M. Van de Klundert, C. de Rooij and L.C. van der Marel, Phys. Comm. Twente Univ. of Techn. 3 (1972) no. 3 .

[3] R.B. Dingle, Physica 19 (1953) 311.

[4] G.E.H. Reuter and E.H. Sondheimer, Proc. Roy. Soc. A195 (1948) 336.

[5] K.R. Lyall and J.F. Cochran, Phys.. Rev. 159 (1967) 517.

[6] H.B.G. Casimir and J. Ubbink, Philips Tech. Rev. 28 (1967) 271,300 and 366. 